\title{
Conflito ambiental de uso do solo na bacia hidrográfica do Tapacurá-PE
}

\author{
Environmental conflict of land use in Tapacurá-PE hydrographic basin \\ Conflicto ambiental de uso del suelo en la cuenca hidrográfica Tapacurá-PE
}

Recebido: 10/02/2021 | Revisado: 14/02/2021 | Aceito: 18/02/2021 | Publicado: 27/02/2021

Salatiel Ewen Braga

ORCID: https://orcid.org/0000-0002-3233-5361

Universidade Federal Rural de Pernambuco, Brasil E-mail: salatiel_braga@hotmail.com

Anildo Monteiro Caldas

ORCID: https://orcid.org/0000-0003-1095-5938

Universidade Federal Rural de Pernambuco, Brasil E-mail: monteiro.dtr.ufrpe@gmail.com

Euzonio Rizzi Neto

ORCID: https://orcid.org/0000-0003-2391-1539

Universidade Federal Rural de Pernambuco, Brasil E-mail: euzi.neto@gmail.com

Lucas José de Souza Silva

ORCID: https://orcid.org/0000-0002-9283-5130

Universidade Federal Rural de Pernambuco, Brasil E-mail: lucasjd.souzaifpe@gmail.com

Allana Monique Bezerra Lustosa Perônico ORCID: https://orcid.org/0000-0003-0542-151X Universidade Federal Rural de Pernambuco, Brasil E-mail: allanaperonico@gmail.com

Ricardo Andrade Wanderley

ORCID: https://orcid.org/0000-0003-1043-1544

Universidade Federal Rural de Pernambuco, Brasil E-mail: ricardo.dtr.ufrpe@gmail.com

Pedro Marcos Lira de Freitas

ORCID: https://orcid.org/0000-0002-9158-6865

Universidade Federal Rural de Pernambuco, Brasil

E-mail: pedromarcoslira@gmail.com

Jonatan Roberto de Lima

ORCID: https://orcid.org/0000-0003-2162-566X

Universidade Federal Rural de Pernambuco, Brasil

E-mail: jonatanrobertodl@gmail.com

Carina Raissa Rocha Oliveira da Cunha

ORCID: https://orcid.org/0000-0002-8408-5122

Universidade Federal Rural de Pernambuco, Brasil

E-mail: 3xcarina@gmail.com

Bruna Silva Abreu

ORCID: https://orcid.org/0000-0001-8682-7188

Universidade Federal Rural de Pernambuco, Brasil

E-mail: bruna-s-abreu@hotmail.com

\begin{abstract}
Resumo
A crescente expansão não planejada das atividades agropecuárias sem um levantamento prévio das potencialidades e limitações quanto ao uso do solo, apresenta significante risco de deterioração do meio ambiente. A atividade agropecuária não planejada representa grande risco ambiental, elevando o índice de erosão por ocupar áreas impróprias devido à sua topografia, ou por atividades poluentes que podem prejudicar os ecossistemas adjacentes, seja por assoreamento dos fluxos superficiais de água ou até mesmo por contaminação de agroquímicos. Esta pesquisa objetivou desenvolver um diagnóstico geoambiental para a região da bacia do Tapacurá nas áreas de possível ocorrência de conflito de uso do solo. Utilizando-se cenas de radar da missão SRTM (Shuttle Radar Topography Mission), submetidos a processamento e análises em Sistemas de Informações Geográficas (SIG). Como resultados desta pesquisa, obtiveram-se a delimitação digital da área de interesse no formato vetorial, bem como, os mapas de declividade reclassificados conforme as classes preconizadas pela Embrapa Solos; somando-se a estes foram obtidas as características morfométricas dos compartimentos hidrológicos contidos na área de interesse, a saber: rede de drenagem, comprimento do talvegue principal, perímetro da bacia, somatório dos cursos d’água e a densidade da rede de drenagem. Estes parâmetros foram calculados pelo módulo ARCSWAT, na plataforma ArcGIS 9 da ESRI. Uma vez calculados estes parâmetros, foram confeccionados os mapas de declividade, índice de rugosidade, uso e ocupação do solo atual e potencial para realizar o diagnóstico ambiental de conflito de uso do solo.
\end{abstract}


Palavras-chave: Diagnóstico geoambiental; MDE; Meio ambiente; Morfometria; SIG.

\begin{abstract}
The growing unplanned expansion of agricultural activities without a prior survey of the potential and limitations regarding land use, presents a significant risk of deterioration of the environment. Unplanned agricultural activity represents a great environmental risk, increasing the rate of erosion by occupying inappropriate areas due to its topography, or by polluting activities that can harm adjacent ecosystems, either by silting up surface water flows or even by contamination of agrochemicals. This research aimed to develop a geoenvironmental diagnosis for the region of the Tapacurá basin in areas of possible occurrence of land use conflict. Using radar scenes from the SRTM (Shuttle Radar Topography Mission) mission, submitted to processing and analysis in Geographic Information Systems (GIS). As a result of this research, the digital delimitation of the area of interest in the vector format was obtained, as well as the slope maps reclassified according to the classes recommended by Embrapa Solos; adding to these, the morphometric characteristics of the hydrological compartments contained in the area of interest were obtained, namely: drainage network, length of the main thalweg, perimeter of the basin, sum of the water courses and the density of the drainage network. These parameters were calculated by the ARCSWAT module, on ESRI's ArcGIS 9 platform. Once these parameters were calculated, the slope maps, roughness index, use and occupation of the current soil and potential to carry out the environmental diagnosis of land use conflict were made.
\end{abstract}

Keywords: Geoenvironmental diagnosis; MDE; Environment; Morphometry; GIS.

\title{
Resumen
}

La creciente expansión no planificada de las actividades agrícolas sin un estudio previo del potencial y las limitaciones en cuanto al uso de la tierra, presenta un riesgo significativo de deterioro del medio ambiente. La actividad agrícola no planificada representa un gran riesgo ambiental, aumentando la tasa de erosión al ocupar áreas inapropiadas por su topografía, o por actividades contaminantes que pueden dañar los ecosistemas adyacentes, ya sea por sedimentación de los caudales superficiales o incluso por contaminación de agroquímicos. Esta investigación tuvo como objetivo desarrollar un diagnóstico geoambiental para la región de la cuenca del Tapacurá en áreas de posible ocurrencia de conflicto de uso de suelo. Utilizando escenas de radar de la misión SRTM (Shuttle Radar Topography Mission), sometidas a procesamiento y análisis en Sistemas de Información Geográfica (GIS). Como resultado de esta investigación, se obtuvo la delimitación digital del área de interés en formato vectorial, así como los mapas de taludes reclasificados según las clases recomendadas por Embrapa Solos; sumado a estos, se obtuvieron las características morfométricas de los compartimentos hidrológicos contenidos en el área de interés, a saber: red de drenaje, longitud del desagüe principal, perímetro de la cuenca, suma de los cursos de agua y la densidad de la red de drenaje. Estos parámetros fueron calculados por el módulo ARCSWAT, en la plataforma ArcGIS 9 de ESRI. Una vez calculados estos parámetros, se realizaron los mapas de taludes, índice de rugosidad, uso y ocupación del suelo actual y potencial para realizar el diagnóstico ambiental de conflicto de uso de suelo.

Palabras clave: Diagnóstico geoambiental; MDE; Medio ambiente; Morfometria; GIS.

\section{Introdução}

A necessidade de promover o desenvolvimento industrial, comercial e econômico de um estado, minimizando impactos ambientais, além de proporcionar os direitos adquiridos da população para bem-estar físico, mental e social, criar mecanismos de desenvolvimento sustentável, que acima de tudo, respeitem as condições locais de cada região. O espaço Estadual caracteriza-se por apresentar uma dinâmica complexa, em constantes e profundas mudanças. Nesta área, diferentes interesses, de diversos agentes técnicos e políticos, disputam regiões sem muitas vezes terem noção dos impactos causados no ambiente.

Desta maneira, uma boa administração deve dispor de um plano estratégico, o qual deve ser apoiado no conhecimento mais preciso possível do espaço de intervenção, para que as atividades de desenvolvimento se tornem eficientes. A crescente expansão das atividades agropecuárias e a urbanização desordenada, sem considerar as potencialidades e limitações quanto ao uso das terras, constitui fonte potencial de deterioração do meio ambiente (Santos, 1994).

No processo de ocupação do espaço e das mudanças no uso do solo ocorre os interesses das gestões políticas administrativas, econômicas, sociais e ambientais. Assim, a partir das atividades antrópicas, a cobertura vegetal natural se modifica na paisagem e os conflitos ocorrem, principalmente quando o uso atual difere do uso potencial, e quando o uso atual está associado às alterações do uso da terra acima da sua capacidade de suporte ou estão em áreas de preservação, conforme a legislação ambiental brasileira. Neste processo, encontram-se envolvidos os problemas de unidades territoriais em qualquer 
esfera administrativa, que ocorrem em lugares, formas e intensidades distintas; demandando ações direcionadas para equacionar esses problemas de forma efetiva sob pena de desperdiçar recursos públicos (Santos, 1994).

Para Moura (2003), a análise ambiental na gestão pública, além de complexa, é um problema notadamente espacial. Daí o emprego do geoprocessamento como instrumento auxiliar aos gestores para aquisição, manipulação, armazenamento, combinação, análise e recuperação de informações importantes que direcionam à tomada de decisões.

O Sistema de Informação Geográfica (SIG) é uma ferramenta poderosa e deve ser encarada como atividade estratégica e rotineira para o processo administrativo. No Brasil, poucas são as prefeituras que utilizam as técnicas de geoprocessamento há mais de uma década (Câmara e Medeiros, 1998; Davis Jr, 2002).

As informações geradas, a partir de mapas temáticos obtidos por técnicas de geoprocessamento para o ordenamento do território são inúmeras, tais como: formações básicas do relevo, hidrografia, características geológicas, geomorfológicas, declividades ou áreas de ocorrências de doenças e área de expansão urbana, análise de infraestrutura, informações socioeconômicas, plano diretor, lei de uso e ocupação do solo, zoneamento, análises e mapeamento de riscos ambientais (Caldas, 2018; Camâra et al., 1996).

Áreas ocupadas por agricultura requerem maior atenção do poder público no que concerne à tomada de decisão de medidas de prevenção, educação pública e reordenamento ocupacional do solo (Caldas, 2015).

O presente trabalho teve como objetivos realizar o diagnóstico geoambiental das áreas de conflito de uso do solo, possibilitando orientar as diretrizes de planejamento territorial, identificando áreas de conflitos de uso da terra para adoção de práticas conservacionistas, promovendo o melhor uso de área no local e viabilizar maior produção respeitando as condições naturais do meio ambiente.

\section{Metodologia}

\section{1 Área de estudo}

O estudo, de caráter quali-quantitativo conforme Pereira (2018), foi realizado na bacia do rio Tapacurá (Figura 1), afluente do rio Capibaribe. Essa bacia está localizada na zona de transição entre a Zona da Mata e o Agreste, no Estado de Pernambuco (Xavier, 2017).

A bacia hidrográfica do rio Tapacurá conta com doze sub-bacias sendo as principais formadas pelos riachos Itapessirica, Natuba, Gameleira e Várzea do Una abrangendo seis municípios, Vitória de Santo Antão, Pombos, São Lourenço da Mata, Gravatá, Moreno e Chã Grande (Dantas, 2011). 
Figura 1. Localização da bacia do rio Tapacurá em relação aos Municípios Pernambucanos.

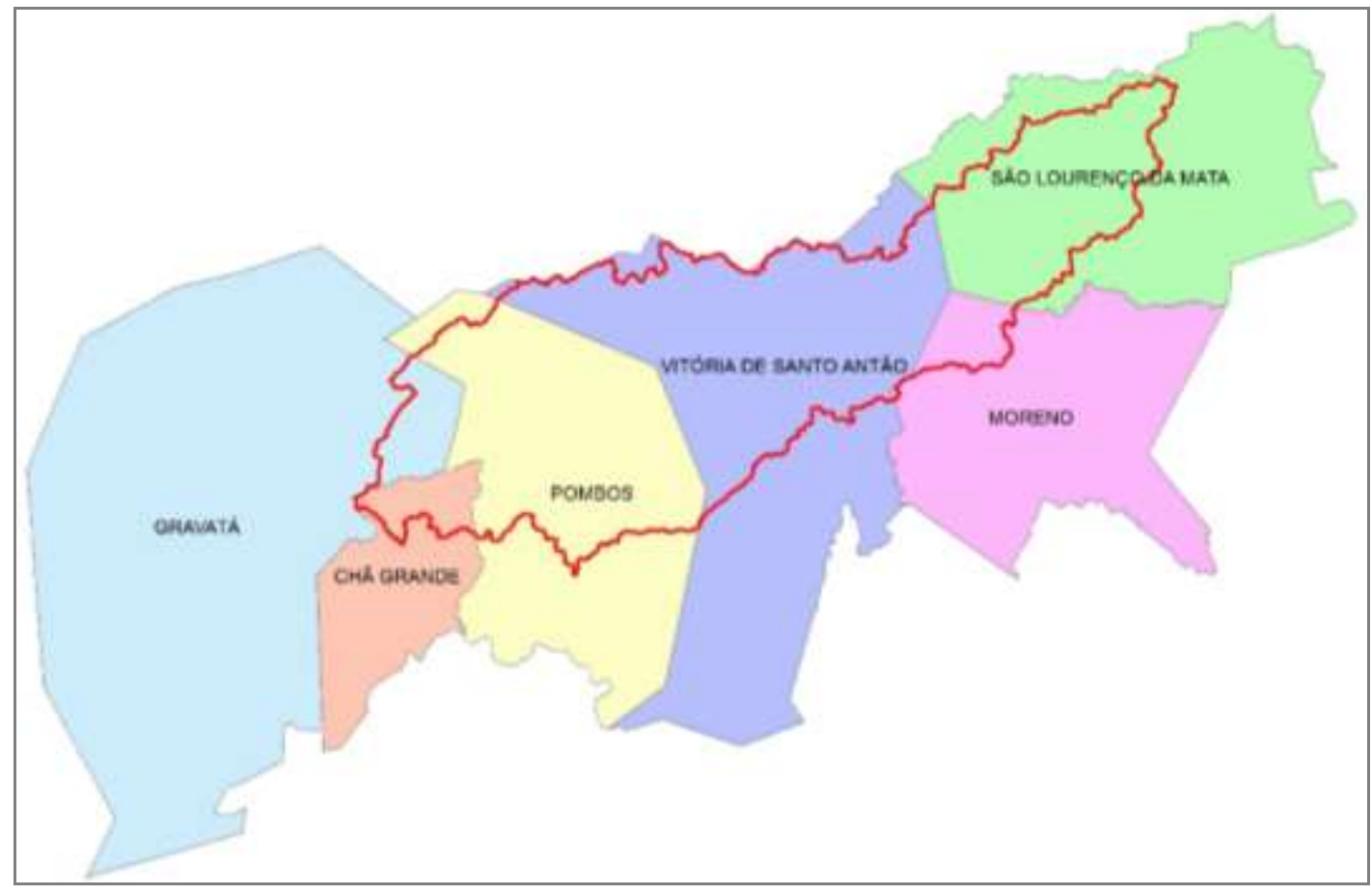

Fonte: Autores.

Na década de 1970, na bacia do rio Tapacurá, no território pertencente ao município de São Lourenço da Mata foi construído o reservatório Tapacurá, com as finalidades de abastecimento para população da região metropolitana do Recife e para o controle de enchentes no rio Capibaribe, evitando assim inundações na cidade do Recife. O rio Capibaribe nasce no município de Poção em Pernambuco, no município de Limoeiro se torna um rio perene, e em São Lourenço da Mata tem o encontro das águas com o rio Tapacurá. O reservatório Tapacurá atende as cidades de Camaragibe, Jaboatão dos Guararapes, Recife e São Lourenço da Mata, contribuindo com aproximadamente $40 \%$ do volume distribuído na região metropolitana e possui capacidade máxima de acumulação de 94,2 milhões de m³ (Braga et al., 2020; Santos, 2010).

O período chuvoso da bacia ocorre entre os meses de março a julho, com temperatura média mensal oscilando entre 23 e $27^{\circ} \mathrm{C}$, enquanto a umidade relativa do ar, durante os meses de maio a agosto é superior a 75\% (Duarte, 2009; Oliveira, 2012).

\subsection{Base de dados e modelagem digital}

Foram utilizados para compor a base do mapeamento e para coleta de dados cenas de imagens de radar SRTM (Shuttle Radar Topography Mission), de resolução espacial de 30 m, e mosaico de imagens de sensor óptico Landsat 8, com resolução espacial de 30 m e resolução temporal de 16 dias, ambos no Datum WGS84, disponíveis gratuitamente nos sites do Instituto Nacional de Pesquisas Espaciais (INPE) e Earth Explorer do United States Geological Survey (USGS). Após a aquisição e montagem do banco de dados, as imagens foram transformadas para projeção UTM (Universal Transversal Mercator) no Datum SIRGAS 2000 (Sistema de Referência Geocêntrico para as Américas).

A modelagem digital foi realizada no software ArcGIS da ESRI GIS and Mapping Software, licenciado para o laboratório de Geotecnologias do Departamento de Tecnologia Rural da UFRPE. O módulo ArcCatalog do ArcGIS, foi utilizado para criação de uma plataforma de armazenamento e organização dos dados e produtos (mapas temáticos) obtidos, denominada GeodataBase "Diagnostico_bacia_tapacura", subdividida em topográficos e morfométricos, uso e conflito. 
A extensão ARCSWAT (Soil and Water Assessment Tool) desenvolvido pelo Blackland Research Center da Texas Agricultural Station e USDA Agricultural Research Service, em trabalhos realizados no Agricultural Research Service e na Texas $A \& M$ University, foi utilizada como ferramenta pra modelagem hidrográfica e obtenção dos compartimentos hidrológicos da bacia.

\subsection{Mapas temáticos}

A metodologia empregada para confecção dos mapas temáticos uso potencial do solo, uso e ocupação atual do solo e conflito de uso do solo, foi a descrita por Rocha e Kurtz, (2001); Valle Junior, (2008); Valle Junior et al., (2013); Valle Junior et al., (2014) e Caldas, (2015).

Os mapas dos fatores ambientais de rede de drenagem, classes de declividade e delimitação da bacia hidrográfica foram obtidos do mosaico SRTM pelo processamento em plataforma ArcMap, bem como os resultados morfométricos dos compartimentos hidrológicos oriundos de processamento em plataforma ARCSWAT.

A elaboração do mapa de uso potencial do solo foi realizada com base no coeficiente de rugosidade (Ruggdeness Number - RN), quanto maior for o valor do RN, maior potencialidade de ocorrer o processo erosivo do solo. Sendo este um parâmetro que direciona o uso potencial das terras, quanto às suas aptidões para atividades de agricultura, pecuária e ao reflorestamento ou para preservação permanente. Na Tabela 1, apresenta-se a classificação do indicativo de uso potencial do solo e respectivos pesos do RN.

Tabela 1. Classificação de aptidão de uso do solo segundo o RN*.

\begin{tabular}{ccc}
\hline Classificação segundo o RN & Indicativo de uso potencial do solo & Peso \\
\hline Aptidão A & Agricultura & 1 \\
Aptidão B & Pecuária & 2 \\
Aptidão C & Pecuária / Floresta & 3 \\
Aptidão D & Floresta & 4 \\
\hline
\end{tabular}

* Ruggdeness Number (Coeficiente de rugosidade). Fonte: Autores.

Para a determinação do coeficiente de rugosidade foi utilizada a equação $\mathrm{RN}=\mathrm{D}$ x H; sendo, $\mathrm{RN}$ o coeficiente de rugosidade, D a densidade da rede de drenagem $\left(\mathrm{km}^{\mathrm{k}} \mathrm{km}^{-2}\right)$ e $\mathrm{H}$ a declividade média (\%) da área de estudo, descrita em Rocha e Kurtz, (2001).

A área da bacia foi dividida em 31 compartimentos hidrológicos, sobre os quais, foram realizadas as análises morfométricas, área $\left(\mathrm{km}^{2}\right)$, comprimentos totais da rede de drenagem $(\mathrm{km})$, densidade de drenagem $\left(\mathrm{km} \cdot \mathrm{km}^{-2}\right)$ e declividade média (\%) como o auxílio da extensão ARCSWAT do ArcGIS, que deram subsídios para o cálculo do RN de cada compartimento hidrológico.

De posse dos valores dos RN, utilizou-se os cálculos estatísticos da amplitude total (diferença entre o valor máximo e o valor mínimo o RN) e do intervalo de classes (amplitude total dividida por 4, onde 4 é número de classes de aptidão do RN), para agrupar os valores de RN nas classes de aptidão de uso do solo.

Para determinar os limites de cada classe, considerou-se como limite inferior da primeira classe (Aptidão A) o menor valor do $\mathrm{RN}$ e como limite superior, somou-se a este o valor do intervalo de classe. Os limites inferiores das classes subsequentes foram fixados a partir dos limites superiores das classes antecedentes, sempre somando a estes o intervalo de classe para obter os limites superiores. 
O mapa de uso e ocupação atual do solo foi gerado a partir do banco de dados pertencente ao IBGE Geociências (2001). Cada classe foi rotulada de acordo com a ocupação do solo, para esse estudo foram consideradas três classes de uso do solo, sendo atribuída a cada uma destas um peso (Tabela 2).

Tabela 2. Classes de uso atual do solo e pesos.

\begin{tabular}{ccc}
\hline Classes & Uso atual do solo & Peso \\
\hline Classe A & Agricultura & 1 \\
Classe B & Pastagem & 2 \\
Classe C & Mata & 3 \\
\hline
\end{tabular}

Fonte: Autores.

O mapa das áreas de conflito de uso foi obtido a partir do método de classificação cruzada, que consiste na superposição de dois planos de informação (álgebra de mapas), a partir da sobreposição dos mapas de uso potencial do solo com o mapa de uso e ocupação atual do solo, possibilitando assim, a elaboração do mapa de conflitos de uso. O procedimento foi processado com o auxílio do software ESRI ArcGIS Desktop, possibilitando a delimitação e classificação das áreas de conflito de uso, segundo a metodologia proposta por Valle Junior, (2008); Valle Junior et al., (2013); Valle Junior et al., (2014) e Caldas, (2015).

Como exemplo, se o peso de RN (Tabela 1) for indicativo de uso para florestas, e atualmente a respectiva área é usada para agricultura (Tabela 2), após a classificação cruzada $(4-1=3$ ) pode-se definir a existência de um conflito de categoria 3 e assim sucessivamente (Tabela 3). Quando o resultado da classificação cruzada for negativo ou zero, indica área de expansão agrícola ou área sem conflito de uso do solo

Tabela 3. Descrição das categorias de conflito de uso do solo (degradação ambiental).

\begin{tabular}{|c|c|c|}
\hline $\begin{array}{l}\text { Peso da aptidão - } \\
\text { Peso do uso atual }\end{array}$ & $\begin{array}{l}\text { Categorias de } \\
\text { conflito }\end{array}$ & Descrição \\
\hline $\begin{array}{l}\text { Exemplos: } \\
\qquad \begin{array}{r}4-3=1 \\
3-2=1 \\
2-1=1\end{array}\end{array}$ & Categoria 1 & $\begin{array}{l}\text { Terras com riscos ou limitações permanentes severas quando usadas para culturas } \\
\text { anuais e pastagens, seu uso deve ser norteado pela implementação de técnicas conjuntas } \\
\text { de conservação do solo (caráter vegetativo, mecânico). }\end{array}$ \\
\hline $\begin{array}{l}\text { Exemplos: } \\
\qquad \begin{array}{r}4-2=2 \\
3-1=2\end{array}\end{array}$ & Categoria 2 & $\begin{array}{l}\text { Terras impróprias para cultivos intensivos, mas ainda adaptadas para pastagem nativa, } \\
\text { reflorestamento ou preservação ambiental. }\end{array}$ \\
\hline $\begin{array}{l}\text { Exemplo: } \\
\qquad 4-1=3\end{array}$ & Categoria 3 & $\begin{array}{l}\text { Terras impróprias para cultivos intensivos e pastagens, mas ainda adaptadas para } \\
\text { reflorestamento ou preservação ambiental. }\end{array}$ \\
\hline
\end{tabular}




\section{Resultados e Discussão}

Com o intuito de delimitar a área da bacia do rio Tapacurá procedeu-se a caracterização morfométrica, em meio computacional, obtendo-se uma área de $471,71 \mathrm{~km}^{2}$, este valor difere em $1,71 \mathrm{~km}^{2}$ da área obtida nos estudos de Xavier (2017), Braga (2001), Silva et al. (2010), Santos et al. (2014), que foi de $470 \mathrm{~km}^{2}$

A Tabela 4 apresenta as áreas e respectivos percentuais em relação aos municípios que abrangem a bacia do Tapacurá. dos quais o maior percentual $38,2 \%$ da área da bacia estão inseridos na divisão política do município de Vitoria de Santo Antão e o menor percentual encontra-se no município de Moreno com 3,6\%.

Tabela 4: áreas e respectivos percentuais em relação os municípios abrangidos pela bacia do Tapacurá.

\begin{tabular}{cccc}
\hline \multirow{2}{*}{ Município } & Áreas dos municípios & \multicolumn{2}{c}{ Áreas } \\
\cline { 3 - 4 } Gravatá & $\left(\mathrm{km}^{2}\right)$ & $\left(\mathrm{km}^{2}\right)$ & 4,7 \\
Chã Grande & 507,38 & 22,19 & 3,7 \\
Pombos & 84,93 & 17,38 & 30,1 \\
Vitória de Santo Antão & 240,1 & 142,07 & 38,2 \\
Moreno & 336,2 & 180,05 & 3,6 \\
São Lourenço da Mata & 196,18 & 17,16 & 19,7 \\
Total & 262,24 & 92,86 & 100 \\
\hline
\end{tabular}

Fonte: Autores.

Os demais municípios apresentaram os percentuais: Pombos (30,1\%), São Lourenço da Mata (19,7\%), Gravatá $(4,7 \%)$ e Chá Grande (3,7\%), calculados no software ArcGis. Tais valores apresentam pouca diferença quando comparados aos valores obtidos por Dantas (2011), onde Vitória de Santo Antão 38,6\%, Pombos 31,2\%, São Lourenço da Mata 19,8\%, Gravatá 4,3\%, Moreno 3,3\% e Chã Grande 2,8\%

A bacia do rio Tapacurá (Figura 2) compreende uma área de $471,71 \mathrm{~km}^{2}$ com talvegue principal apresentando uma extensão 66,52 km, com padrão formado pelos cursos d'água caracterizando-se como do tipo dendrítico, pois sua rede de drenagem assemelha-se a uma formação arborescente. Segundo Soares (2000), esse padrão ocorre em terras altas, nas quais o regolito e a rocha mãe oferecem uma resistência relativamente uniforme à erosão.

Quanto ao escoamento global, esta microbacia pode ser classificada como exorreica, pois é uma sub-bacia do Rio Capibaribe que desagua no mar. Ainda analisando a Figura 2 constatou-se, segundo a classificação de Strahler (1957), que a bacia apresenta uma ordenação de canais de $4^{\mathrm{a}}$ ordem, condizendo com o relevo forte ondulado característico da bacia. 
Figura 2. Rede de drenagem da bacia do Tapacurá.

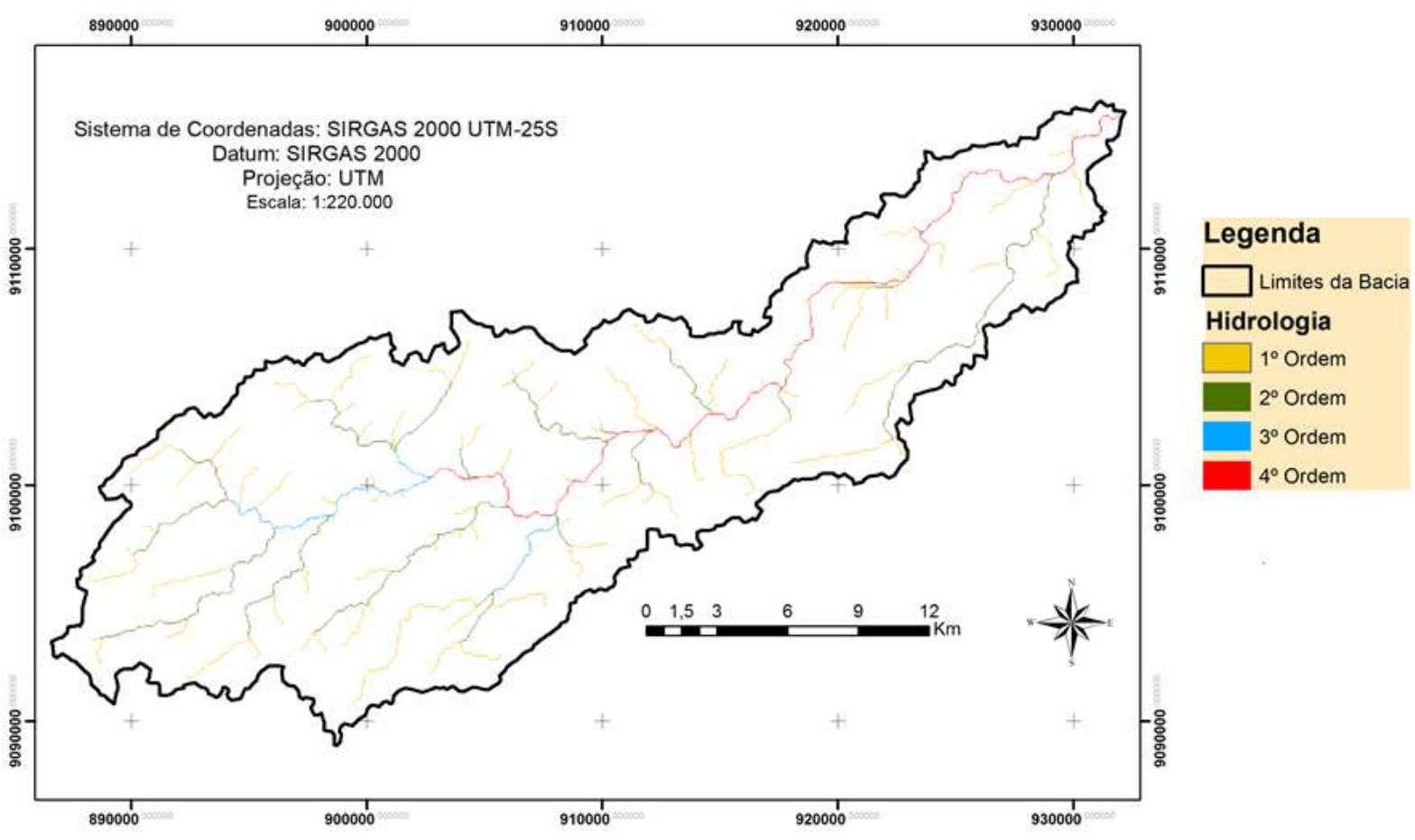

Fonte: Autores.

Com relação ao relevo, cuja classificação é feita em 6 classes de declividade (Figura 3): Plano (0-3\%), Suave Ondulado (3-8\%), Ondulado (8-20\%), Fortemente Ondulado (20-45\%), Montanhoso (45-75\%), Forte-Montanhoso/Escarpado (> 75\%) segundo Embrapa (1979), há uma predominância do relevo ondulado com 42,7\% da área da bacia, este relevo caracteriza-se por apresentar superfície de topografia pouco movimentada, constituída por colinas e/ou outeiros, apresentando declives moderados (Chagas, 2013), e menor percentual de relevo Forte-Montanhoso/Escarpado cerca de $0,001 \%$.

Figura 3: Classes de declividade preconizadas pela EMBRAPA SOLOS da bacia do Tapacurá.

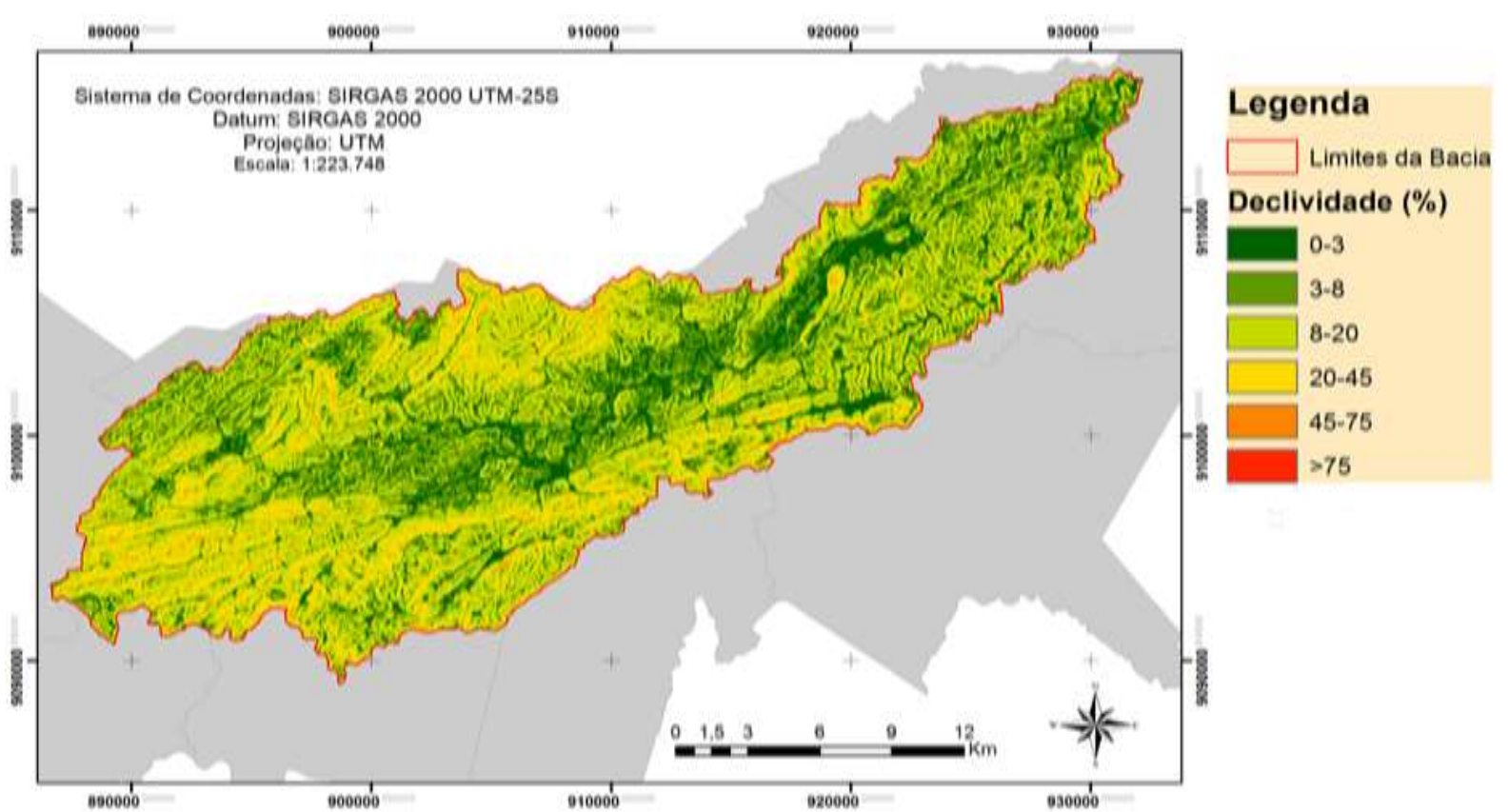

Fonte: Autores. 
O relevo é um fator muito importante em relação ao uso e manejo dos solos, pois, o relevo implica sérias limitações ao ambiente, tornando-o mais suscetível à erosão e impedindo a mecanização. O uso das áreas com relevos suave ondulado e ondulado requer a implementação de práticas simples de conservação de solos, tais como: preparo do solo e plantio em contorno, uso de renques de vegetação permanente em contorno, rotação de culturas e incorporação dos restos culturais ao solo (Campos, 2012 e Saadi, 1995).

A aptidão do uso potencial do solo para cada compartimento hidrológico foi determinada pelo coeficiente de rugosidade (RN), em 4 classes (Tabela 5). O maior e menor valor de RN ocorridos na área foram de 12,18 a 0,46. Calculada a amplitude de variação, obteve-se os valores de amplitude 11,72 e de intervalo de classe de 2,93.

Tabela 5: Intervalos de domínio de RN para classificação da aptidão de uso potencial do solo para a bacia do Tapacurá.

\begin{tabular}{ccc}
\hline Aptidão A & $0,46-3,39$ & Agricultura \\
Aptidão B & $3,39-6,32$ & Pecuária \\
Aptidão C & $6,32-9,25$ & Pecuária/Floresta \\
Aptidão D & $9,25-12,18$ & Floresta \\
\hline
\end{tabular}

*Amplitude de RN: 11,72; Intervalo de classe: 2,93. Fonte: Autores.

A aptidão "A", com indicativo para agricultura, é representada pelos menores valores de RN, em contrapartida, a aptidão "D", com indicativo para floresta é representada pelos maiores valores de RN. Isto se deve ao fato que, quanto maior for o valor do RN entre os compartimentos hidrológicos, maior o perigo de erosão, o que justifica a destinação das áreas com maior RN para áreas de preservação permanentes, uma vez que seu uso por atividades antrópicas ocasionaria a deterioração destes ambientes (Valle Junior et al., 2014). Uma vez determinado os intervalos referentes a cada aptidão de uso, os compartimentos hidrológicos foram classificados dentro da aptidão correspondente aos seus respectivos valores de RN e pesos conforme a Tabela 6. 
Tabela 6: Atributos dos compartimentos hidrológicos da bacia do Tapacurá.

\begin{tabular}{|c|c|c|c|c|c|c|c|}
\hline $\mathbf{C H} \mathbf{H}^{*}$ & Área** & $\% * * *$ & $\begin{array}{l}\text { Declividade } \\
\text { média***** }\end{array}$ & $\begin{array}{c}\text { Densidade de } \\
\text { drenagem } * * * * *\end{array}$ & $\begin{array}{c}\text { Coeficiente de } \\
\text { rugosidade-RN }\end{array}$ & $\begin{array}{c}\text { Classificação de } \\
\text { RN }\end{array}$ & Peso \\
\hline $\mathrm{CH} 01$ & 11,09 & 2,39 & 9,05 & 0,45 & 4,08 & Aptidão B & 2 \\
\hline $\mathrm{CH} 02$ & 31,61 & 6,81 & 9,15 & 0,33 & 3,06 & Aptidão A & 1 \\
\hline $\mathrm{CH} 03$ & 12,54 & 2,70 & 11,15 & 0,04 & 0,46 & Aptidão A & 1 \\
\hline $\mathrm{CH} 04$ & 14,78 & 3,18 & 10,88 & 0,62 & 6,70 & Aptidão C & 3 \\
\hline $\mathrm{CH} 05$ & 15,65 & 3,37 & 11,28 & 0,09 & 1,00 & Aptidão A & 1 \\
\hline $\mathrm{CH} 06$ & 47,25 & 10,17 & 12,67 & 0,33 & 4,19 & Aptidão B & 2 \\
\hline CH 07 & 6,07 & 1,31 & 9,48 & 0,64 & 6,08 & Aptidão B & 2 \\
\hline CH 08 & 14,99 & 3,23 & 14,82 & 0,11 & 1,66 & Aptidão A & 1 \\
\hline CH 09 & 15,60 & 3,36 & 18,06 & 0,30 & 5,34 & Aptidão B & 2 \\
\hline CH 10 & 5,40 & 1,16 & 11,19 & 0,40 & 4,49 & Aptidão B & 2 \\
\hline $\mathrm{CH} 11$ & 15,39 & 3,31 & 12,31 & 0,24 & 2,94 & Aptidão A & 1 \\
\hline CH 12 & 26,21 & 5,64 & 14,63 & 0,19 & 2,28 & Aptidão A & 1 \\
\hline CH 13 & 9,85 & 2,12 & 21,31 & 0,1 & 2,20 & Aptidão A & 1 \\
\hline CH 14 & 13,22 & 2,85 & 14,45 & 0,18 & 2,64 & Aptidão A & 1 \\
\hline $\mathrm{CH} 15$ & 1,72 & 0,37 & 10,17 & 1,20 & 12,18 & Aptidão D & 4 \\
\hline CH 16 & 18,83 & 4,05 & 10,21 & 0,12 & 1,18 & Aptidão A & 1 \\
\hline CH 17 & 15,94 & 3,43 & 12,79 & 0,31 & 2,90 & Aptidão B & 2 \\
\hline CH 18 & 8,09 & 1,74 & 6,82 & 0,54 & 3,69 & Aptidão B & 2 \\
\hline CH 19 & 0,07 & 0,02 & 4,84 & 1,77 & 8,56 & Aptidão C & 3 \\
\hline $\mathrm{CH} 20$ & 14,15 & 3,05 & 10,96 & 0,38 & 4,14 & Aptidão B & 2 \\
\hline CH 21 & 4,14 & 0,89 & 7,00 & 0,55 & 3,87 & Aptidão B & 2 \\
\hline CH 22 & 10,05 & 2,16 & 20,02 & 0,03 & 0,68 & Aptidão A & 1 \\
\hline $\mathrm{CH} 23$ & 14,07 & 3,03 & 16,59 & 0,26 & 4,30 & Aptidão B & 2 \\
\hline $\mathrm{CH} 24$ & 10,46 & 2,25 & 15,49 & 0,32 & 5,02 & Aptidão B & 2 \\
\hline $\mathrm{CH} 25$ & 2,39 & 0,51 & 9,93 & 1,10 & 10,92 & Aptidão A & 1 \\
\hline CH 26 & 8,41 & 1,81 & 16,20 & 0,60 & 9,79 & Aptidão A & 2 \\
\hline CH 27 & 35,54 & 7,65 & 15,22 & 0,24 & 3,65 & Aptidão A & 3 \\
\hline CH 28 & 14,16 & 3,05 & 14,10 & 0,20 & 2,83 & Aptidão A & 4 \\
\hline CH 29 & 23,51 & 5,06 & 20,01 & 0,29 & 5,87 & Aptidão B & 2 \\
\hline CH 30 & 28,32 & 6,10 & 21,12 & 0,24 & 5,17 & Aptidão B & 2 \\
\hline CH 31 & 14,97 & 3,22 & 17,44 & 0,18 & 3,21 & Aptidão A & 1 \\
\hline
\end{tabular}

*CH: compartimento hidrológico; **Área: $\mathrm{km}^{2} ; \% * * *$ : Percentual em relação à área total da bacia; ****Declividade: \%; *****Densidade de drenagem: $\mathrm{km} / \mathrm{km}^{2}$. Fonte: Autores.

Observa-se, na Tabela 6, que o compartimento hidrológico de maior extensão territorial foi classificado com aptidão B, com indicativo para pastagem, com área de $47,25 \mathrm{~km}^{2}$ e o menor compartimento hidrológico com área de $1,72 \mathrm{~km}{ }^{2}$ obteve indicativo para floresta (aptidão D). Dos 31 compartimentos hidrológicos 15 apresentaram aptidão para agricultura, 13 para pecuária, 2 para pecuária/floresta e 1 para floresta o que denota grande potencial agrícola da bacia.

Quando somadas as áreas dos compartimentos hidrológicos, da bacia do Tapacurá (Tabela 7), pertencentes a mesma classe de aptidão de uso potencial, obteve-se 243,81 km² para agricultura (aptidão A), 204,09 km² para pecuária (Aptidão B), $22,09 \mathrm{~km}^{2}$ para pecuária/floresta (aptidão C) e $1,72 \mathrm{~km}^{2}$ para floresta (aptidão D). 
Tabela 7: Áreas dos indicativos de uso potencial do solo da bacia do Tapacurá.

\begin{tabular}{ccc}
\hline Indicativo de uso potencial do solo & Áreas $\left(\mathrm{km}^{2}\right)$ & $\%{ }^{*}$ \\
\hline Aptidão A - Agricultura & 243,82 & 52,49 \\
Aptidão B - Pecuária & 204,07 & 43,94 \\
Aptidão C - Pecuária/Floresta & 14,85 & 3,2 \\
Aptidão D - Floresta & 1,72 & 0,37 \\
\hline Total & $464,56^{* *}$ & 100 \\
\hline
\end{tabular}

* Percentual em relação à área total da bacia. ** Área diferente da apresentada pelo IBGE visto que o ARCSWAT desconsidera algumas áreas que não apresentam relevância para quantificação da rede de drenagem. Fonte: Autores.

A partir dos valores dos pesos do RN adquiridos na Tabela 6, obteve-se o mapa da distribuição espacial do uso potencial do solo para a bacia do rio Tapacurá (Figura 4). Como o RN é um parâmetro ambiental que direciona o uso potencial das terras que implica na suscetibilidade do solo à erosão, na Figura 4, é possível constatar as áreas mais suscetíveis à erosão classificadas nas classes de aptidões C e D.

Figura 4: Mapa de uso potencial do solo da bacia do Tapacurá.

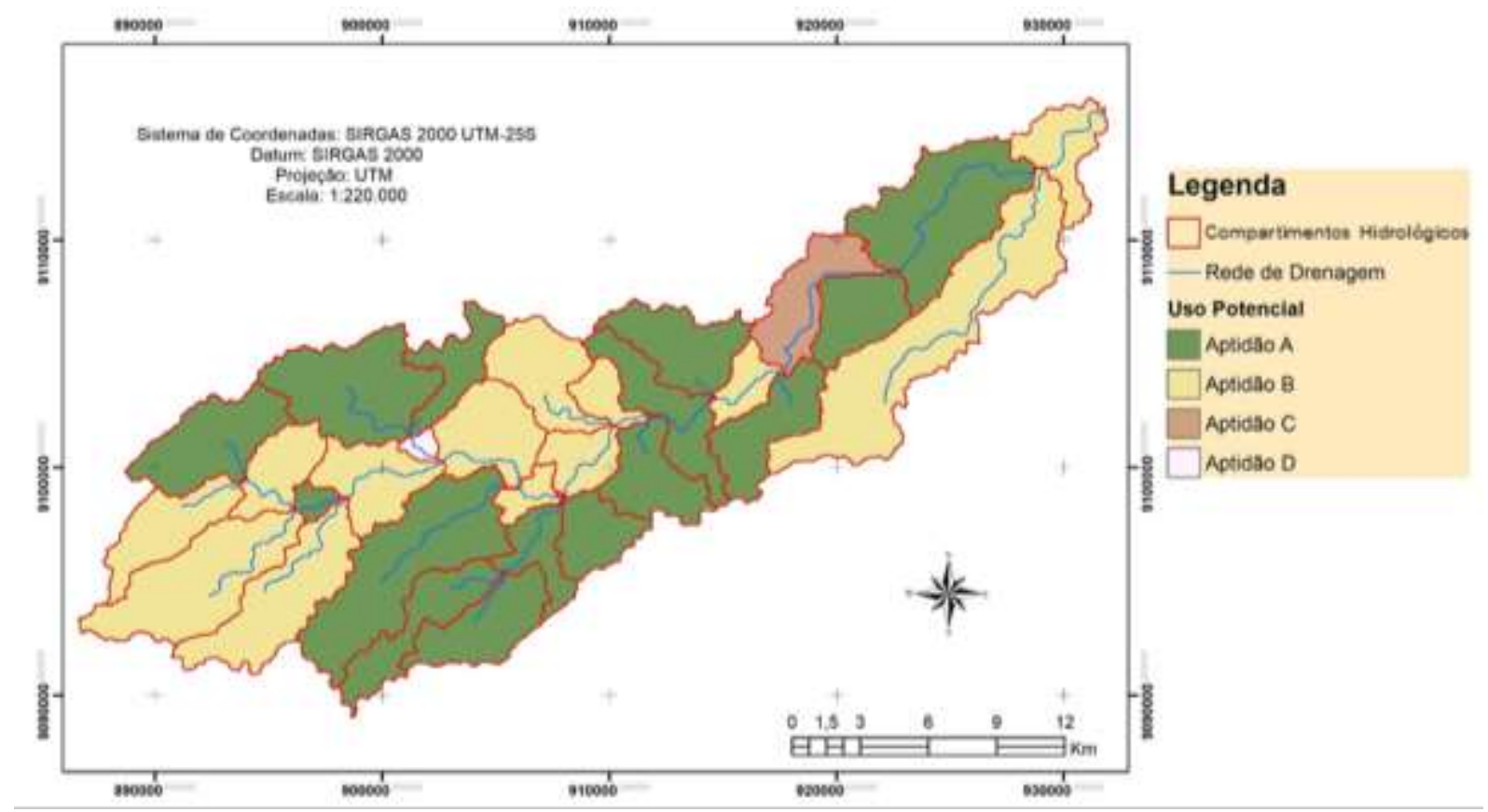

Fonte: Autores.

Sampaio et al. (2010) afirmaram que capacidade de uso da terra e RN se equivalem em 99,9\% dos casos e, assim, propôs a substituição da capacidade de uso da terra pelo RN, por ser um método mais rápido e sem necessidade de coleta de amostras no campo, facilitando principalmente, o aspecto econômico.

O uso atual do solo identificado na bacia do Tapacurá apresentou a área de $390,94 \mathrm{~km}^{2}$ de agricultura, $12,42 \mathrm{~km}^{2}$ de mata, $46,2 \mathrm{~km}^{2}$ de área de pastagem e 15,46 km² de área urbana que equivale a 82,7\%, 2,63\%, 9,77\% e 3,27\% da área da bacia do Tapacurá, respectivamente (Tabela 8). 
Tabela 8: Áreas dos usos atuais do solo da bacia do Tapacurá.

\begin{tabular}{ccc}
\hline Uso Atual & Áreas $\left(\mathrm{km}^{2}\right)$ & $\%^{*}$ \\
\hline Agricultura & 390,94 & 82,7 \\
Mata & 12,42 & 2,63 \\
Corpos Hídricos & 7,71 & 1,63 \\
Pastagem & 46,2 & 9,77 \\
Área urbana & 15,46 & 3,27 \\
\hline Total & 472,72 & 100 \\
\hline
\end{tabular}

* Percentual em relação à área total da bacia. Fonte: Autores.

Para análise de uso do solo é constituído objeto da cobertura vegetal, as ações antrópicas e os ambientes naturais nativos da região. Nestas áreas nativas aponta-se a medida para a preservação do patrimônio natural, que preconiza-se pela legislação ambiental brasileira. O mapa da Figura 5 demonstra a distribuição espacial do uso e ocupação do solo da bacia do Tapacurá, considerando as classes de pastagem, agricultura, mata e urbana.

Figura 5: Mapa de uso atual do solo da bacia do Tapacurá.

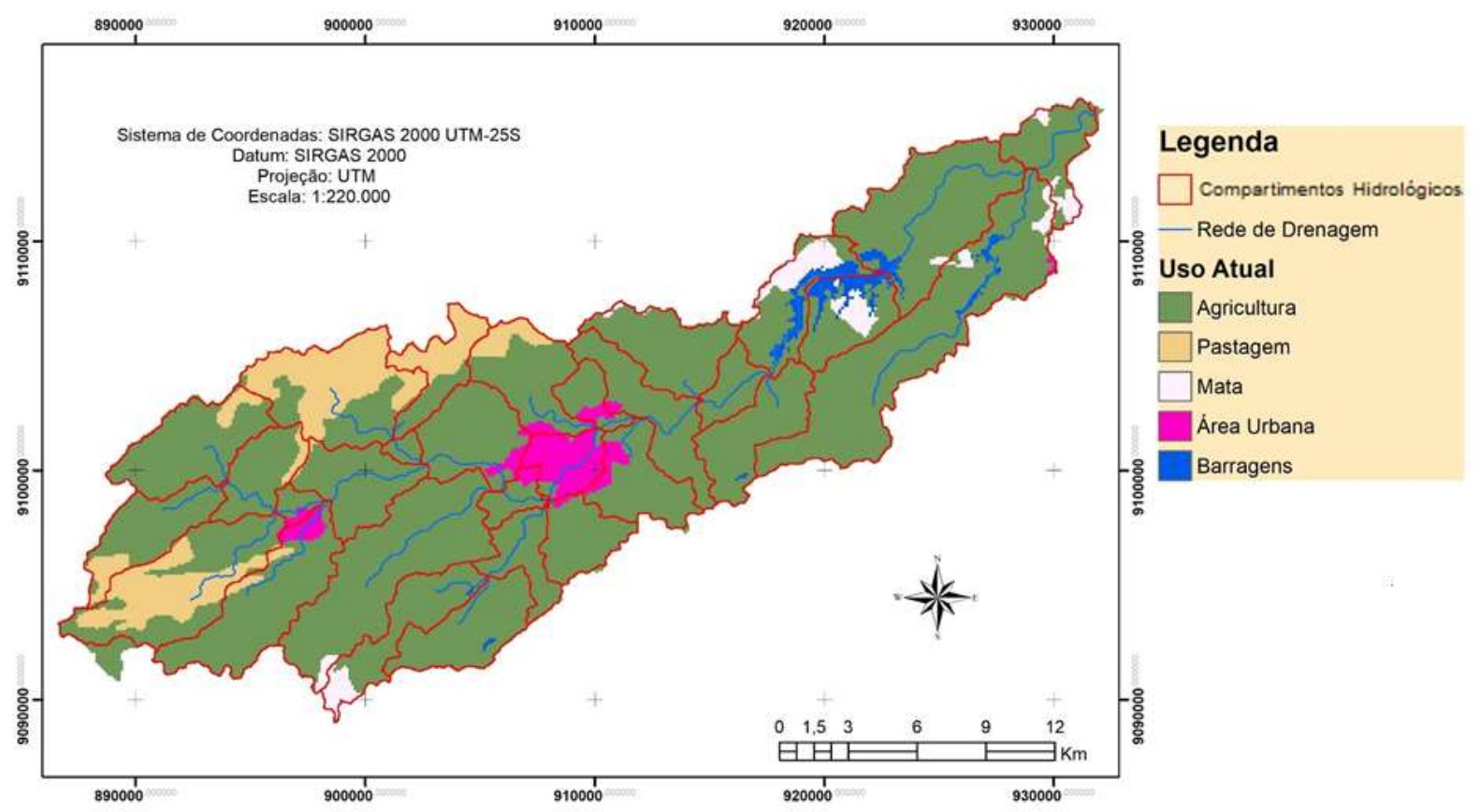

Fonte: Autores.

A classe de uso mais expressiva é representada pela agricultura, com maior área para a cultura de cana de açúcar. Esta cultura é a principal matéria prima para a fabricação do açúcar e etanol, fazendo parte da base econômica da região. O cultivo geralmente é feito de forma extensiva e as plantações ocupam muitas áreas contíguas. Como o cultivo e o corte são realizados por máquinas e tratores, o manejo do solo ocorre de forma intensa. Concomitantemente à produção, o manejo deverá ser conservacionista.

Outra superfície de destaque são as áreas de pastagem. Este sistema apresenta longos períodos de ocupação do solo com animais. Apesar da preocupação dos produtores em melhorar a utilização dos produtos forrageiros, o manejo adequado não ocorre. E, um dos principais problemas desse sistema é a falta de um manejo conservacionista, que normalmente culmina com a degradação do solo. 
A distribuição espacial das áreas das categorias de conflito de uso na área da bacia do Tapacurá é apresentada na Figura 6. Um conflito pode existir, mas não necessariamente gerar um processo erosivo antrópico. Entretanto, ressalta-se a importância da implantação adequada das práticas de manejo nas atividades agropecuárias na região.

Figura 6: Mapa de conflito de uso do solo na bacia do Tapacurá.

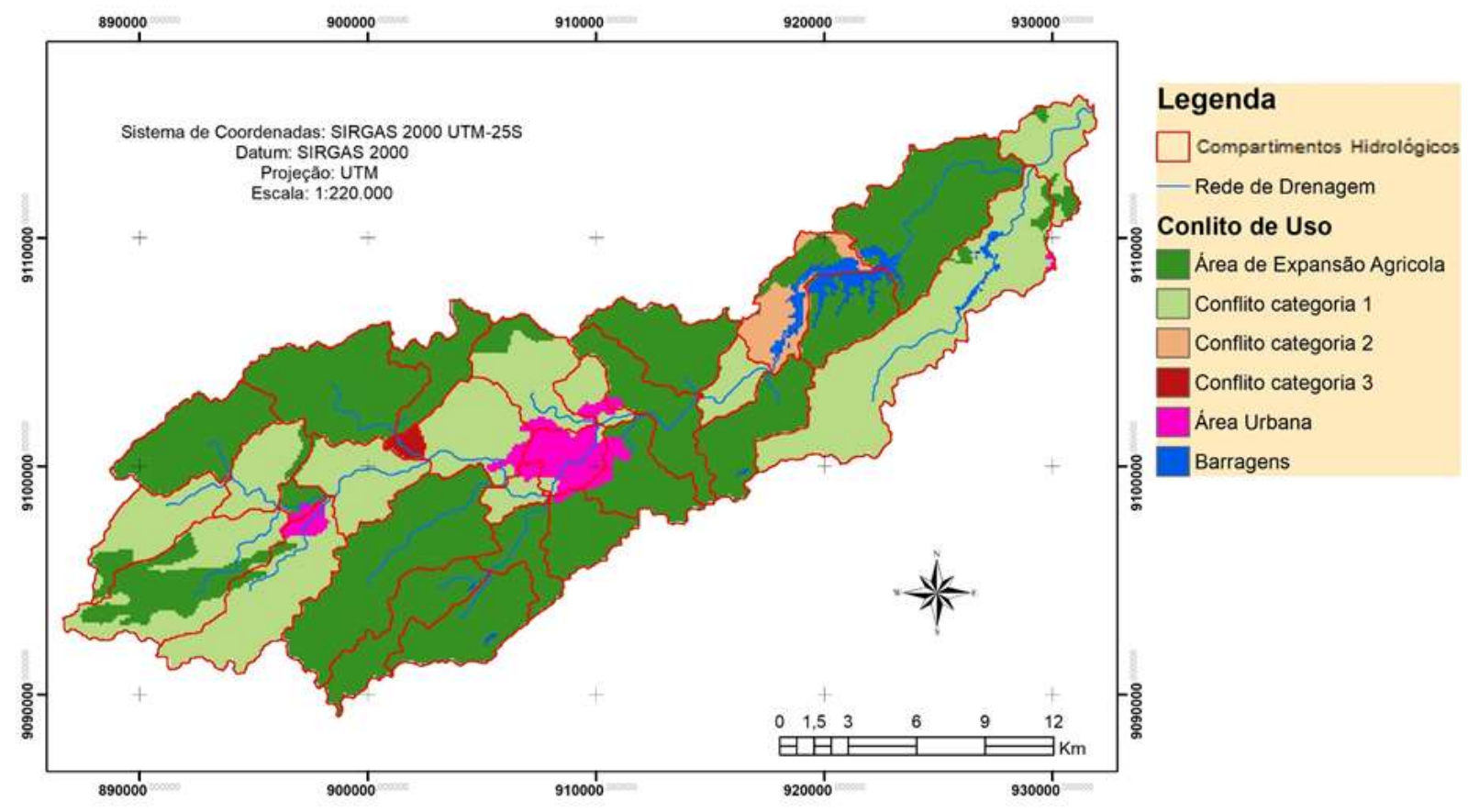

Fonte: Autores.

$\mathrm{Na}$ Tabela 9, encontram-se os valores em $\mathrm{km}^{2}$ e respectivos percentuais das três categorias de conflito identificadas. Dos 471,71 km² da bacia, 176,82 km² encontram-se em zonas de conflito. A classe de conflito 1, que são áreas que apresentam riscos ou severas limitações de forma permanente, quando utilizadas para culturas de periodicidade anual e pastagens, devem ser norteadas pela implementação de técnicas conjuntas de conservação do solo, ocupam a maior área da bacia do, com 166,53 $\mathrm{km}^{2}$ ou $35,23 \%$.

* Percentual em relação à área total da bacia. Fonte: Autores.

Tabela 9: Área das categorias de conflito de uso da bacia do Tapacurá.

\begin{tabular}{ccc}
\hline Classe de Conflito & Áreas $\left(\mathrm{km}^{2}\right)$ & $\% *$ \\
\hline Categoria 1 & 166,53 & 35,23 \\
Categoria 2 & 8,59 & 1,82 \\
Categoria 3 & 1,71 & 0,36 \\
\hline Total & 176,82 & 37,41
\end{tabular}

A classe de conflito 2, que representa as terras inapropriadas à cultivos intensivos, porém adaptadas à pastagem nativa, reflorestamento ou preservação ambiental, compreende 8,59 km2, correspondem em 1,82\% da área da bacia. Nessas áreas, recomendam-se práticas conservacionistas atentando para a importância de reduzir a perda de solo e, consequentemente, aumentar o fornecimento de nutrientes para as plantas. O grande potencial dos sistemas florestais é uma estratégia para conservação dos solos (Candido et al., 2010).

Ainda na Tabela 9, cerca de 1,71 km2 encontra-se na classe de conflito 3 , correspondente às terras inapropriadas à 
cultivos intensivos e pastagens, porém adaptadas ao reflorestamento ou preservação ambiental, representando 0,36\% da área da bacia.

Segundo Campos et al. (2009), as áreas de preservação permanentes têm papel vital dentro de uma região, por serem responsáveis pela manutenção, preservação e conservação dos ecossistemas existentes. Assim, estas devem estar sempre cobertas com a vegetação original, pois a cobertura vegetal minimiza os efeitos erosivos e a lixiviação dos solos, contribuindo também para regularização do fluxo hídrico, redução do assoreamento dos cursos d’água e reservatórios, trazendo benefícios diretos para a fauna e para os seres humanos.

A análise efetuada possibilitou compreender que a origem desses conflitos é a ocupação inadequada, por conta de ações antrópicas, e, em muitos casos, de ocupações irregulares com manejo inadequado, sem se considerar a legislação ambiental brasileira.

É importante salientar que as legislações federais, estaduais e municipais resguardam os recursos naturais existentes na área e que a região deveria adotar planejamentos para o uso e ocupação do solo, respaldados em estudos como o apresentado, bem como rigorosa fiscalização visando a preservação e manutenção dos recursos naturais para as próximas gerações.

\section{Considerações Finais}

A caracterização morfométrica da área de estudo através do modelo SWAT, possibilitou o melhor entendimento da área, bem como possibilitou a obtenção de informações relevantes para o processo de uso e ocupação da bacia do rio Tapacurá.

A análise da classe de conflitos mostra que o conflito classe 1 , referentes às terras que apresentam riscos ou severas limitações de forma permanente, quando utilizadas para culturas de periodicidade anual e pastagem, representa a maior parte com 166,53 km2 da Bacia.

A classe de conflito 2, que representa as terras inapropriadas à cultivos intensivos, porém adaptadas à pastagem nativa, reflorestamento ou preservação ambiental, compreende $8,59 \mathrm{~km} 2$, correspondendo a $1,82 \%$ da área total da bacia.

A classe de conflito 3 , correspondente às terras inapropriadas à cultivos intensivos e pastagens, porém adaptadas ao reflorestamento ou preservação ambiental, compreende $1,71 \mathrm{~km} 2$, representando $0,36 \%$ da área total da bacia.

Este trabalho evidencia o quão necessário se faz a verificação das limitações quanto aos usos da terra, que podem alterar o equilíbrio dinâmico das características naturais da paisagem e comprometer o sistema ambiental e a qualidade de vida do homem. Assim, é necessário conhecer melhor a dinâmica dessa área para se estabelecer diretrizes que subsidiem a utilização, manejo, medidas conservacionistas dos recursos naturais e adoção de restrições mais seguras quanto ao usoe manejo do solo.

O uso inadequado do solo atua na fragilidade e a na potencialidade do ambiente integrada ao uso e ocupação antrópica do solo. Nestes casos, as áreas estão ocupadas inadequadamente, o que demanda sua readequação.

Recomenda-se um planejamento das atividades de implantação de sistemas de produção em consonância com o meio, e determinação de áreas prioritárias no que tange à sua ordenação e reordenação.

Evidenciou-se no estudo que a utilização do solo sem considerar sua aptidão potencial ocasiona conflitos que geram impactos ambientais, sendo necessário a realização de estudos futuros que visem a identificação desses impactos tais como, riscos de inundação e vulnerabilidade à erosão ocasionados pelo uso e ocupação indevidos do solo.

\section{Referências}

Braga, R. A. P. (2001). Gestão ambiental da bacia do Rio Tapacurá-Plano de ação. Universitária da UFPE. 
Braga, S. E., Wanderley, R. A., Holanda, T. F. de, \& Caldas, A. M. (2020). Aplicação da Análise Hierárquica para mapeamento de risco de inundação: O caso no município de Limoeiro (Pernambuco - Brasil). Meio ambiente (Brasil), 2(1), 57-66.

Caldas, A. M. (2015). Diagnóstico geoambiental no município de Batatais-SP. (Tese de Doutorado, Universidade Estadual Paulista Júlio de Mesquita Filho, Faculdade de Ciências Agrárias e Veterinárias de Jaboticabal).

Caldas, A. M., Pissarra, T. C. T., Costa, R. C. A., Neto, F. C. R., Zanata, M., Parahyba, R. D. B. V., \& Pacheco, F. A. L. (2018). Flood vulnerability, environmental land use conflicts, and conservation of soil and water: A study in the Batatais SP municipality, Brazil. Water, $10(10), 1357$.

Camâra, G., \& Medeiros, J. D. (1998). Princípios básicos em geoprocessamento. Sistemas de informações geográficas: aplicações na agricultura, 2, 3-11.

Câmara, G., Casanova M. A., Hemerly, A. S., Magalhães, G. C., \& Medeiros, C. M. B. (1996). Anatomia de Sistemas de Informação Geográfica. Instituto de Computação, UNICAMP. Campinas. 193p.

Campos, M. C. C. (2012). Relações solo-paisagem: conceitos, evolução e aplicações. Ambiência. Guarapuava (PR) 8(3), 963 - 982.

Campos, S., Pessoa, M. L., Barbosa, A. P., Pissarra, T. C. T., \& Mora, V. B. (2009). Geoprocessamento aplicado na identificação e localização potencial de conflitos de uso em áreas de preservação permanente na microbacia do córrego Monte Belo, Botucatu (SP, Brasil). Acta Agronómica, 58(4), $299-302$.

Candido, H. G., Galbiatti, J. A., Pissarra, T. C., \& Martins Filho, M. V. (2010). Degradação ambiental da bacia hidrográfica do Rio Uberaba: uma abordagem metodológica. Engenharia Agrícola, 30(1), 179-192.

Chagas, C. S., Fontana, A., Carvalho Junior, W. de, \& Caires, S. M. de. (2013). Atributos topográficos na diferenciação de argissolos. Revista Brasileira de Ciências do solo. 37(6), 1441-1453.

Coutinho Duarte, C. (2009). Análise dos impactos das mudanças climáticas no escoamento superficial da bacia hidrográfica do rio Tapacurá-PE, a partir da utilização de um modelo de balanço hídrico mensal semidistribuído (Dissertação de Mestrado, Universidade Federal de Pernambuco).

Dantas, R. R. L., Tavares, R. G., \& Silva, V. de P. (2011). Poluição da Bacia do Rio Tapacurá: Floração de Cianobactérias, Consequência do Nível Trófico. XIV IWRA Word Water Congress, Ipojuca, PE.

Davis Junior, C. A. (2002). Geoprocessamento: dez anos de transformações. IP: Informática Pública, 4(1), 17-24.

IBGE (2001). Manuais Técnicos em Geociências: Introdução ao Processamento Digital de Imagens. Rio de Janeiro, Brasil: Instituto Brasileiro de Geografia e Estatística.

EMBRAPA. (1979). Serviço Nacional de Levantamento e Conservação de Solos (Rio de Janeiro, RJ). Manual de métodos de análise de solo. Rio de Janeiro. $1 \mathrm{v}$

Moura, A. C. M. (2003). Geoprocessamento na gestão e planejamento urbano: Ed. da autora.

Oliveira, L. M. M. (2012). Estimativa da evapotranspiração real por sensoriamento remoto na bacia do Rio Tapacurá - PE. (Tese de Doutorado, Universidade Federal de Pernambuco).

Pereira A. S. et al. (2018). Metodologia da pesquisa científica. Ed. UAB/NTE/UFSM

Rocha, J. S. M., \& Kurts, S. M. J. M. (2001) Manual de manejo integrado de bacias hidrográficas. (4a ed.): UFSM/CCR. 120 p.

Saadi, A. A. Geomorfologia da Serra do Espinhaço em Minas Gerais e de suas margens. Geonomos, 3(1):41-63, 1995.

Sampaio, M. V., Santos, M. S., Rocha, J. S. M., Paula, M. D, \& Mendes, A. V. (2010). Deterioração físico-conservacionista da sub-bacia hidrográfica do Rio Ibicuí-Mirim - RS. Ciência e Agrotecnologia, Lavras, 34(2), 300-306.

Santos, J. D., Silva, R. D., Montenegro, S., \& Santos, C. (2014). Aplicação do modelo SWAT para a estimativa da produção de sedimentos na bacia do rio Tapacurá, Pernambuco. XI Encontro Nacional de Engenharia de Sedimentos. João Pessoa, PB.

Santos, L. C. C. (2010). Estimativa de vazões máximas de projeto por modelos determinísticos e probabilísticos. (Dissertação de Mestrado, Universidade Federal do Espírito Santo).

Santos, M. (1994). Metamorfose do espaço urbano: Hucitec.

Silva, R. M. D., Silva, L. P., Montenegro, S. M. G. L., \& Santos, C. A. G. (2010). Análise da variabilidade espaço-temporal e identificação do padrão da precipitação na bacia do rio Tapacurá, Pernambuco. Sociedade \& Natureza, 22(2), 357-372.

Soares, J. V. (2000). Curso de introdução à hidrologia florestal. INPE. 78p.

Strahler, A. N. (1957). Quantitative analisys of watershed geomorphology. New Haven: Transamerican Geophysics University, 38,913 - 920.

Valle Junior, R. F. D. (2008). Diagnóstico de áreas de risco de erosão e conflito de uso dos solos na bacia do rio Uberaba. (Tese de Doutorado, Faculdade de Ciências Agrárias e Veterinárias, Universidade Estadual Paulista "Júlio de Mesquita Filho").

Valle Junior, R. F., Galbiatti, J. A., Pissarra, T. C. T., \& Martins Filho, M. V. (2013). Diagnóstico de conflito de uso e ocupação do solo na bacia do Rio Uberaba. Global Science and Technology, Rio Verde-GO, 6, 40-52.

Valle Junior, R. F., Varandas, S. G. P., Sanches Fernandes, L. F., \& Pacheco, F. A. L. (2014). Environmental land use conflicts: a threat to soil conservation. Land use policy, 41, 172-185. 
Research, Society and Development, v. 10, n. 2, e52310212833, 2021

(CC BY 4.0) | ISSN 2525-3409 | DOI: http://dx.doi.org/10.33448/rsd-v10i2.12833

Xavier, A. P. C. (2017). Modelagem temporal da dinâmica do uso e ocupação do solo nas perdas e deposição de sedimentos na bacia do rio Tapacurá (PE). (Dissertação de Mestrado, Universidade Federal da Paraíba). 